\title{
LASER SCANNER TECHNOLOGY, GROUND-PENETRATING RADAR AND AUGMENTED REALITY FOR THE SURVEY AND RECOVERY OF ARTISTIC, ARCHAEOLOGICAL AND CULTURAL HERITAGE
}

\author{
V. Barrile ${ }^{\mathrm{a}}$, G. Bilotta ${ }^{\mathrm{b}, *}$, G.M. Meduri ${ }^{\mathrm{a}}$, D. De Carlo ${ }^{\mathrm{a}}$, A. Nunnari ${ }^{\mathrm{a}}$ \\ ${ }^{a}$ Geomatics Lab, DICEAM, Università Mediterranea di Reggio Calabria, 89123 loc. Feo di Vito, Reggio Calabria, Italy - \\ vincenzo.barrile@unirc.it, ing.giuseppemariameduri@gmail.com, domenico.decarlo@unirc.it, ninonunnari@hotmail.it \\ b Dept. of Planning, IUAV University of Venice, Santa Croce 191, Tolentini 30135 Venice, Italy - \\ giuliana.bilotta@iuav.it
}

KEY WORDS: Laser Scanner, Ground Penetrating Radar, Cultural heritage, Survey, Augmented Reality

\begin{abstract}
:
In this study, using technologies such as laser scanner and GPR it was desired to see their potential in the cultural heritage. Also with regard to the processing part we are compared the results obtained by the various commercial software and algorithms developed and implemented in Matlab. Moreover, Virtual Reality and Augmented Reality allow integrating the real world with historical-artistic information, laser scanners and georadar (GPR) data and virtual objects, virtually enriching it with multimedia elements, graphic and textual information accessible through smartphones and tablets.
\end{abstract}

\section{INTRODUCTION}

The development of new detection technology has allowed great strides to acquire spatial data for various applications. Among them, the scanner technology is one method that allows rapid and comprehensive capture of data and with the help of GPR is possible to analyze the ground below and surrounding the structure (Akca and Gruen, 2007), (Barazzetti, Remondino, Scaioni, 2010), (Barrile et al., 2016). The application of this particular technique and interesting finds its importance in the cultural and architectural context especially in the survey of buildings, statues and artefacts. The survey carried out to the church of "Our Lady of the Poor" (church "Pepe"), located in the city of Reggio Calabria, it is a good example, because this church is a religious building dating back to the tenth century built with a Byzantine style, also its location should be part of the acropolis of the city of Reggio Calabria. The equipment used consists of a Faro Laser Scanner Focus3D, which allowed the survey of internal and external surfaces of the church with a high density of points, supported by a photographic survey carried out by the instrument itself. Using this tool, we have highlighted the noninvasiveness of the investigative technique used and the speed of acquisition that has allowed realizing the survey in a few hours of work and with an easy positioning of the instrument.

The next step is the reconstruction of the structure of the scan data to obtain the 3D model in high definition (Cuca et al. 2011), (Barrile, Meduri, Bilotta, 2011), (Barrile, Bilotta, Pozzoli, 2017),. It can be seen from different angles and allows the creation of educational films or use the data for other platforms such as AutoCAD or Architectural software in case in which there was the need to perform interventions of the conservation of the good.

As for the study of the flooring, we used the GPR performing scans with different frequencies, which are useful to understand what you may be under the floor because the building's history, we think that there is the presence of a crypt or the presence of the three apses they made up the tenth century church.

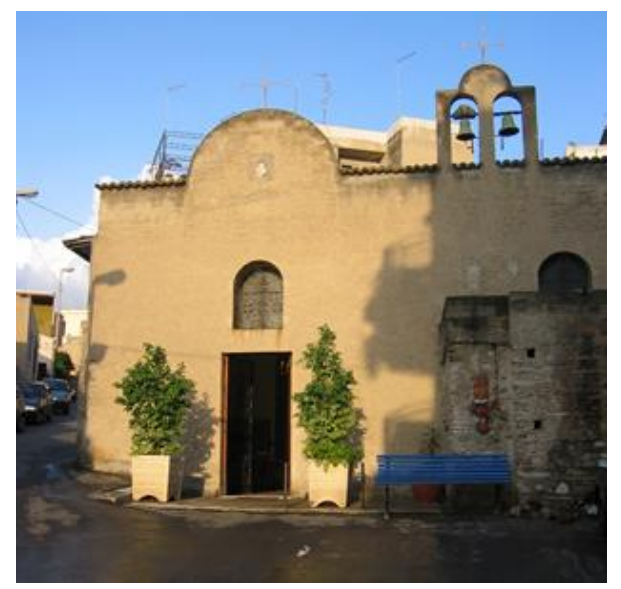

Figure 1. Madonna dei Poveri's Church (chiesa Pepe), Reggio Calabria

\section{LASER SCANNER SURVEY}

The Laser Scanner is a very important tool when you are in a cultural context as through its non-invasiveness allows a large amount of the asset in question without damaging it, and then make the damage that could affect its historical artistic and economic value.

In particular, this study brings to obtain an algorithm to use on Matlab in order to make the alignment. To record the clouds, each time was used the ICP algorithm (Iterative Closest Point) implemented in the Matlab environment.

\footnotetext{
* Corresponding author
} 
The ICP algorithm iteratively applied a rigid roto-translation in one of the two clouds, considered to be mobile, so that overlap in the best possible way to another cloud, considered fixed.

Given a point cloud $\mathrm{V}^{\mathrm{j}}$ and a point cloud $\mathrm{V}^{\mathrm{i}}$ to align with each other, for each yi point of $\mathrm{V}^{\mathrm{j}}$, exists at least one xi point on the cloud $\mathrm{V}^{\mathrm{i}}$, said corresponding point, which is the closest to $\mathrm{y}_{\mathrm{i}}$ compared to all other points in $\mathrm{X}$.

The algorithm is an efficient method to tackle rigid registration between two point sets. Its goal is to find a rigid transformation, with which $\mathrm{Y}$ is registered to be in the best alignment with $\mathrm{X}$, that is, let $\mathrm{T}$ of Equation:

$$
\min _{T, j \in\left\{1,2, \ldots, N_{x}\right\}}\left(\sum_{i=1}^{N_{y}}\left\|T\left(\vec{y}_{i}\right)-\vec{x}_{j}\right\|_{2}^{2}\right)
$$

be rotation and translation transformations, hence the rigid registration between two point sets is

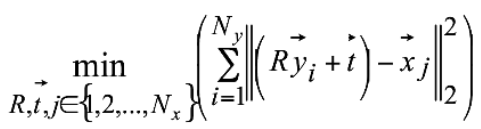

s.t. $\quad \mathrm{R}^{\mathrm{T}} \mathrm{R}=\mathrm{I}_{\mathrm{x}} ; \operatorname{det}(\mathrm{R})=1$

In an iteration, ICP assumes that the closest points correspond, computes the absolute orientation and applies the resulting rigid transformation to $\mathrm{V}^{\mathrm{j}}$. In practice, at step 1 for each point of mobile cloud $\left(\mathrm{V}^{\mathrm{j}}\right.$ set $)$, are sought, within the fixed point cloud, the points (closest point) contained in a sphere of a certain radius (multiple of a parameter introduced by user) belonging to $\mathrm{V}^{\mathrm{i}}$ set. The closest of these will be held and considered the corresponding point.

$$
\left.C_{k}(i)=\underset{j \in\left\{1,2, \ldots, N_{x}\right\}}{\arg \min }\right\}\left(\left\|\left(R_{k-1} \vec{y}_{i}+\vec{t}_{k-1}\right)-\vec{x}_{j}\right\|_{2}^{2}\right)
$$

With these matches found, in step 2, the algorithm computes the incremental transformation (rotation matrix $\mathrm{R}_{\mathrm{i}, \mathrm{j}}$ and translation vector $\mathrm{T}$ and solving the absolute orientation) by applying it to the elements of $\mathrm{V}^{\mathrm{j}}$; If the mean square error is less than a certain threshold, the iteration terminates otherwise return to step 1;

$$
\left(R_{k}, \overrightarrow{t_{k}}\right)=\underset{R^{T} R=I_{m}, \operatorname{det}(R)=1, \vec{t}}{\arg \min }\left(\left\|\left(\overrightarrow{y_{i}}+\vec{t}\right)-\vec{x}_{c_{k}(i)}\right\|_{2}^{2}\right)
$$

The principle on which is based this algorithm is that the alignment between the two point clouds corresponds to the minimization of the quadratic error of the minimum distances between the two objects. In fact, Besl and McKay demonstrated that the algorithm converges to a local minimum of the error.

In this survey, the tool was useful to obtain a $3 \mathrm{D}$ model of the church starting from the acquisition of the scans by positioning the tool both inside and outside the building (Kraus, 2007), (Monti et al., 2004). Then, using the Scene software and JRC Reconstructor is performed the filtering operation eliminating various double points or other types of errors obtained during the survey phase, what is done it is past the alignment of the various scans to obtain so the complete 3D model of the church.

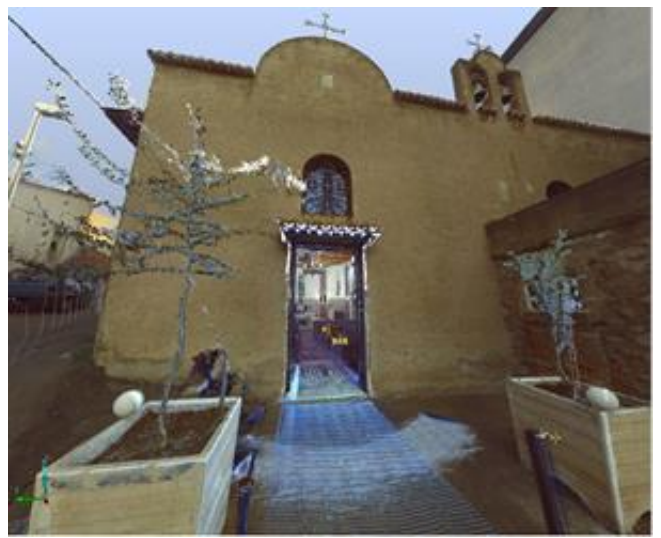

Figure 2. Recostruction 3D of Madonna dei Poveri's church (chiesa Pepe), Reggio Calabria; outside view
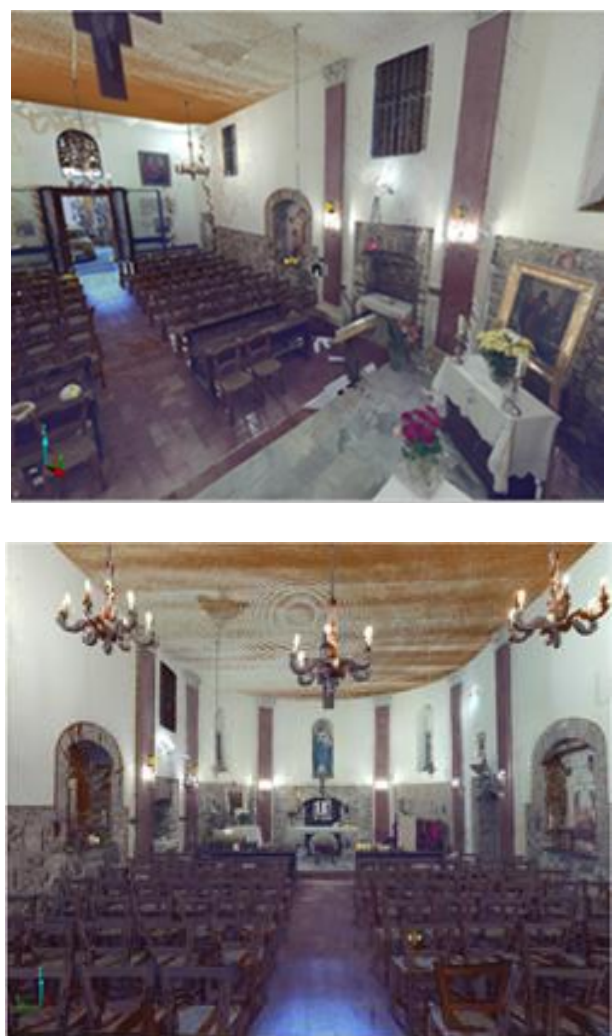

Figures 3,4. Recostruction 3D of Madonna dei Poveri's church (chiesa Pepe), Reggio Calabria; internal view

In addition, the results obtained from the use of this technique can be exploited for example to the disclosure in the tourism sector through the use of the App Webgis or the use of Augmented Reality, or at the architectural level to understand the health status good, or to use in case of renovations or repairs.

\section{GPR SURVEY}

The investigations were carried out using the equipment GPR IDS RIS-K2 made by Systems Engineering SpA, provided with an antenna $200 \mathrm{MHz}$ 's The profiles were acquired in continuous 
mode. The acquisition time window was set at $250 \mathrm{~ns}$ and the sampling time of $0.5 \mathrm{~ns}$.

The GPR data was stored, during the campaign of survey, in .dt format on a special support notebook. A pair of operators carries all of investigation operations out in one day. The data were processed off-line. The processing sequence, then listed, was adopted prior to the commissioning treatment focus:

- $\quad$ First -time alignment

- dewow,

- background removal

- time gain

All of the processing routines are implemented in MatLab 2008b and executed on a PC Support. The algorithm used on Matlab is called Diffraction Summation because when a monostatic GPR moves on a scan line along the $y$ axis, a buried object located in the point of coordinates $\left(\mathrm{y}_{\alpha}, \mathrm{z}_{\alpha}\right)$, gives rise to a hyperbolic track displayed in the a B-scan, described by the following equation:

$$
R_{\alpha}=\sqrt{z_{\alpha}^{2}+\left(y_{\alpha}-y_{\beta}\right)^{2}}
$$

where $\mathrm{R}_{\alpha}$ is the distance between the measuring position $\left(\mathrm{y}_{\beta}, \mathrm{z}=\right.$ $0)$ and the coordinates scatterer buried object $\left(\mathrm{y}_{\alpha}, \mathrm{z}_{\alpha}\right)$.

The technique Diffraction Summation is the simplest approach for focusing the track representing the target for if $\Psi$ is the scalar field representing the data collected along the scan line $y$, the scalar field focused $\Psi_{\mathrm{f}}$ can be obtained as the sum of each point, in the B-scan crude, along the hyperbolic curve of diffraction defined earlier by the equation:

$$
\Psi_{f}\left(y_{\alpha}, z_{\alpha}\right)=\sum_{\beta} \Psi\left(y_{\beta, t}=\frac{R_{\alpha}}{v_{e}}\right)
$$

where $\mathrm{v}=\mathrm{vf} / 2$ is the speed of the waves in the middle obtained by exploiting model "exploding source". If the electromagnetic properties of the soil in which is buried the object vary slowly, it may be used a single velocity $\mathrm{v}$ and for the whole extension in depth (that is, the medium can be considered to be homogenous).

\section{KIRCHHOFF MIGRATION}

The Kirchhoff migration method is based on the same principle characterizing the Diffraction Summation differing, however, for the treatment of "raw data" $\Psi$ collected during the scan through the introduction of some correction factors. The scalar field focused $\Psi_{\mathrm{f}}$ can be described (in discrete form) as:

$$
\Psi_{f}(y, z)=\iint \breve{\Psi}\left(k_{y}, \omega\right) e^{j\left(k_{z}(\omega) z+k_{y} y\right)} d k_{y} d \omega
$$

where $\mathrm{k}_{\mathrm{y}}$ and $\mathrm{k}_{\mathrm{z}}$ are the wave numbers, $\omega$ is the angular pulsation and $\left(\mathrm{k}_{\mathrm{y}}, \omega\right)$ is the Fourier transform of $\Psi(\mathrm{y}, \mathrm{t})$. Taking into account that the wave number $\mathrm{k}_{\mathrm{z}}$ is given by:

$$
k_{z}=\sqrt{\left(\frac{\omega}{v_{e}}\right)^{2}-k_{y}^{2}}
$$

Consequently:

$$
\Psi_{f}(y, z)=v_{e}^{2} \iint \frac{k_{z}}{\omega} \breve{\Psi}\left(k_{y}, \omega\right) e^{j\left(k_{z} y+k_{z} z\right)} d k_{y} d k_{z}
$$

The report is computationally significant: it allows to get the data from focusing $\Psi_{\mathrm{f}}(\mathrm{y}, \mathrm{z})$ as simply the inverse Fourier transform of the product between $\mathrm{k}_{\mathrm{z}} / \omega$ and the two-dimensional Fourier transform of the raw data.

\section{SAR MIGRATION}

The SAR migration technique can be derived considering a scenario in which are buried $\mathrm{N}$ scatterers, each with reflectivity $\rho_{\alpha}$ and coordinates $\left(\mathrm{y}_{\alpha}, \mathrm{z}_{\alpha}\right)$ (for $\left.\alpha \hat{\mathrm{I}}\{1, \ldots, \mathrm{N}\}\right)$. As a result, $\Psi$ (y, t) can be modeled as:

$$
\Psi_{f}(y, z)=\sum_{\alpha=1}^{\alpha=N} \rho_{\alpha} \exp \left(-j \frac{\omega}{v_{e}} \sqrt{z_{\alpha}^{2}+\left(y_{\alpha}-y\right)^{2}}\right)
$$

When you consider the Fourier transform and apply the stationary phase method, it can be shown that the relationship can be expressed as follows:

$$
\breve{\Psi}\left(k_{z}, k_{y}\right)=P(\omega) \sum_{\alpha=1}^{\alpha=N} \rho_{\alpha} \exp \left(-j z_{\alpha} k_{z}+j y_{\alpha} k_{y}\right)
$$

where $\mathrm{P}(\omega)$ is the Fourier transform of the impulse GPR and $\mathrm{k}_{\mathrm{z}}$ is provided by the report. If the GPR image focused $\Psi_{\mathrm{f}}(\mathrm{y}, \mathrm{z})$ is taken as:

$$
\Psi_{f}=\sum_{\alpha} \rho_{\alpha} \delta\left(y-y_{\alpha}, z-z_{\alpha}\right)
$$

You obtained:

$$
\Psi_{f}=\iint \frac{\breve{\Psi}\left(k_{y}, \omega\left(k_{z}\right)\right)}{P\left(\omega\left(k_{z}\right)\right)} \exp \left(-j k_{y} y+j k_{z} z\right) d k_{y} d k_{z}
$$

Accurate assessment with an FFT (Fast Fourier Transform) requires equidistant points in the domain $\left(\mathrm{k}_{\mathrm{y}}, \mathrm{k}_{\mathrm{z}}\right)$, which involve a problem.

From the results obtained from the use of this type of approach it can be seen that, below the pavement, there is probably the presence of a scale that could lead to a crypt and everything is possible to observe from the hyperbola obtained already at a height of 2 meters under the ground level.
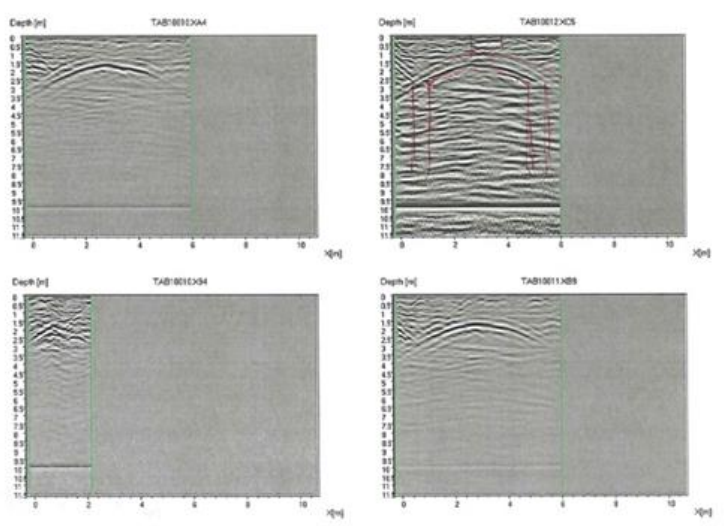

Figure 5. B-scan raw profile (GPR).and B-scan focused using the algorithm Diffraction Summation. 

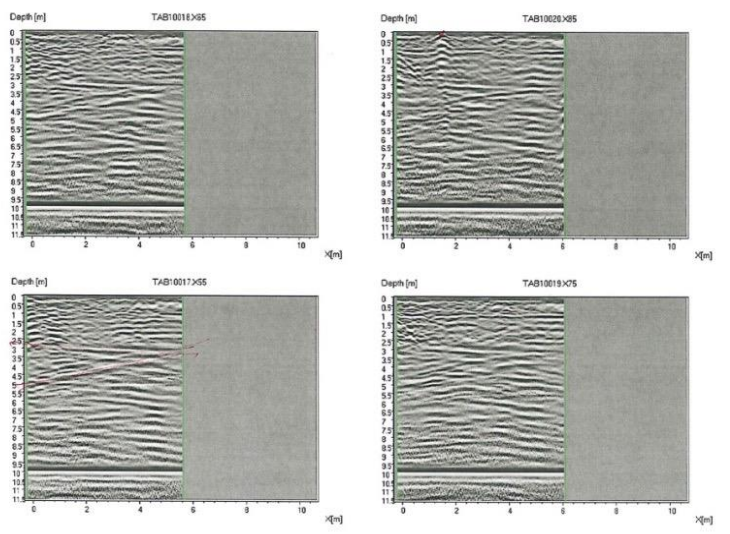

Figure 6. B-scan raw profile (GPR). and B-scan focused using the algorithm Diffraction Summation.

\section{VIRTUAL AND AUGMENTED REALITY}

Virtual Reality is a powerful tool that allows you to recreate and display three-dimensional environments and objects and interact with them in real time providing a sense of immersion and presence in the rebuilt environment. A virtual reconstruction has historical, educational and scientific value as it recreates past environments that are no longer based on historical data. Its information enables to dive into the past to investigate and study and can also be used to assess over time the possible deterioration of the same environments by comparing 3D reconstructions obtained using images that portray the same environments at different times.

Therefore, by appropriately integrating laser scanner data with images captured by the instrument during scans, it is possible to create movies in which the users can immerse themselves within the $3 \mathrm{~d}$ virtual reconstruction of the church and explore it while not being physically present inside it.

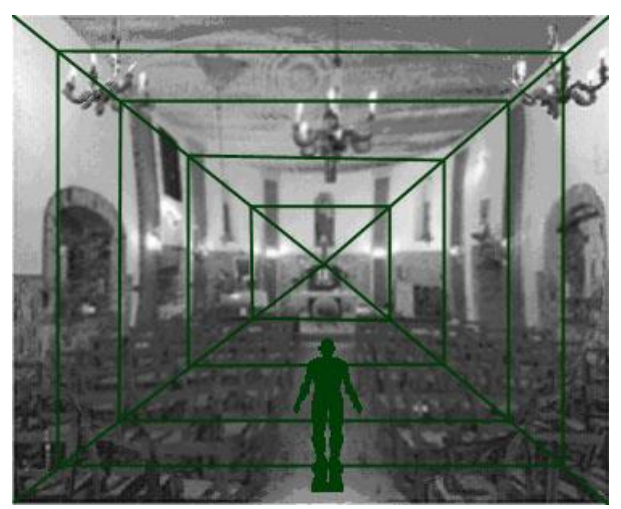

Figure 7. Virtual Reality.

An emerging technology through the spread of ever-rich portable devices with potential content and applications, the daughter of Virtual Reality is the Augmented Reality (Bae et al., 2013).

It consists in adding additional information to the actual or virtually recreated scene; however, unlike Virtual Reality that tends to replace the real world with a completely synthetic environment and isolated from the real one, Augmented Reality integrates the real world with information and virtual objects.
The real world is enhanced, "augmented", virtually enriched, with virtual and multimedia elements, geolocated data, graphic and textual information, etc.

Even with this tool, experiments have been carried out with the aim of making all historical-artistic information, laser scanners and georadar (GPR) data, images and movies accessible through smartphones and tablets equipped with geolocation and internet connection systems. For example, using a mobile phone can frame the surrounding environment in real time; Real-world content levels - Information, Points of Interest (POIs), images, movies and any results from TLS and georadar surveys - are overlapped to the real world - subdivided by typology.

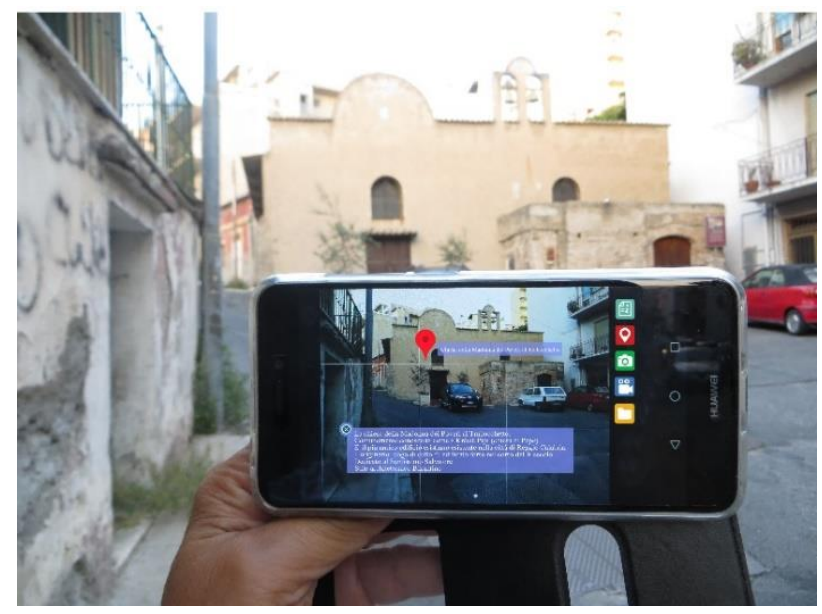

Figure 8. Augmented Reality information.

\section{CONCLUSIONS}

The use of these two instruments shows that the study of the architectural and cultural heritage can be approached very easily without causing any damage. Moreover, the use of the abovementioned Laser Scanner allows obtaining a realistic 3D model and making the community aware of the cultural value of its territory. This can be done by enhancing the heritage itself through simple and easily available applications, that show the characteristics of the monument and its $3 \mathrm{D}$ view. More attention should be placed on its use in the case of monuments subject to conservation and restoration work, since by means of previous monitoring, it is always possible, to bring them back to their original state without ruining them.

The Laser Scanner is particularly interesting when GPR is used because it enables to "find" all the hidden objects of some artistic and cultural value, and to make them known to the community. Finally, the comparison between the software processing and the algorithms developed showed that there are no differences in the results obtained, through the GPR while the 3D model processing showed sub-centimetric differences between the algorithm and the software processing.

We are working to improve the results obtained using programs implemented in our own, thus deepening the research that seems to give promising results. 


\section{ACKNOWLEDGEMENTS}

Our acknowledgements here for Dr. Rosa C. Ponterio, Institute for Chemical-Physical Processes of CNR - Messina.

\section{REFERENCES}

Akca, D., and A. Gruen. 2007. Generalized least squares multiple 3D surface matching. ISPRS WS Laser Scanning 2007 [cd rom], vol. XXXVI, part 3 /W52, Espoo Finland, pp. 1-7.

Bae, H., Golparvar-Fard, M., White, J., 2013. High-precision vision-based mobile augmented reality system for context-aware architectural, engineering, construction and facility management (AEC/FM) applications. Visual. Eng. 1(1), pp. 1-13.

Barazzetti, L., Remondino, F., Scaioni M., 2010. Orientation and 3D modelling from markerless terrestrial images: Combining accuracy with automation, Photogrammetric Record 25(132), pp. 356-381.

Barrile, V., Meduri, G.M., Bilotta, G., 2009. Laser scanner surveying techniques aiming to the study and the spreading of recent architectural structures. In: Recent Advances in Computer Engineering, Proceedings of the 9th WSEAS International Conference on Signal, Speech and Image Processing, SSIP '09, Budapest. pp. 92-95.

Barrile, V., Meduri, G.M., Bilotta, G., 2011. Laser scanner technology for complex surveying structures. Wseas transactions on signal processing, Vol. 7, pp. 65-74.

Barrile, V., and Bilotta, G., 2014. Self-localization by Laser Scanner and GPS in automated surveys. Computational Problems in Engineering. Lecture Notes in Electrical Engineering, Springer, Vol. 307, pp. 293-313.

Barrile, V., Meduri, G.M., Bilotta, G., 2014a. Experimentations and Integrated Applications Laser Scanner/GPS for Automated Surveys. Wseas transactions on signal processing, Vol. 10, pp. 471-480.

Barrile, V., Meduri, G.M., Bilotta, G., 2014b. Comparison between Two Methods for Monitoring Deformation with Laser Scanner. Wseas transactions on signal processing, Vol. 10, pp. 497-503.

Barrile, V., Bilotta, G., D’Amore, E., Meduri, G.M., Trovato, S., 2016. Structural Modeling of a Historic Castle Using Close Range Photogrammetry. International Journal of Mathematics and Computers in Simulation, Vol. 10, pp. 370-380.

Barrile, V., Bilotta, G., Meduri, G.M., 2016. Identifying damage on cars through the integrated use of TLS/SfM with thermographic images. International Journal of Circuits, Systems and Signal Processing, Vol. 10, pp. 433-439.

Barrile, V., Bilotta, G., Pozzoli, A., 2017. Comparison between innovative techniques of photogrammetry. In: The 2016 International Conference Applied Mathematics, Computational Science and Systems Engineering, Roma (Italia), 5-7 november 2016, ITM Web of Conferences, Vol. 9, pp. 1-7.

Cuca, B., Brumana, R., Scaioni, M., and Oreni, D., 2011. Spatial data management of temporal map series for cultural and environmental heritage. In: International Journal of Spatial Data Infrastructures Research, IJSDIR Vol. 6, pp 1-31.
Kraus, K., 2007. Photogrammetry-Geometry from images and laser scans. Berlin, Germany: Walter de Gruyter,

Monti, C., Brumana, R., Achille, C., and Fregonese L., 2004. Per un moderno rilievo della basilica di San Lorenzo tra opera, progetto e trasformazioni. ed. Silvana, Milan, Italy, pp. 25-242.

Rinaudo, F., Bornaz, L., and Ardissone, P., 2007. 3D high accuracy survey and modelling for Cultural Heritage Documentation and Restoration. In: Vast 2007-future technologies to empower heritage professionals, November $26-$ 29, 2007, Brighton, UK, Archaeolingua Hun, pp. 19-23. 